\title{
Processing multiple recognition probes in short- and long-term memory
}

\author{
RICHARD C. MOHS and KEITH T. WESCOURT \\ Stanford University, Stanford, California 94305
}

\begin{abstract}
On each trial in a recognition memory task either one or two test probes were presented. If one probe was presented, subjects were to respond yes if it was a member of a well learned long-term memory set or a member of a short-term memory set that was changed on each trial; subjects were to give a no response if the probe was not a member of either memory set. If two probes were presented, subjects were to give a yes response only if both were memory set members and were to give a no response otherwise. Several aspects of the results indicate that multiple probes are processed sequentially in this task.
\end{abstract}

A modified version of the Sternberg (1969) recognition memory paradigm was used by Wescourt and Atkinson (1973) to investigate recognition processes in a task involving both short-term store (STS) and long-term store (LTS). In the modified paradigm subjects memorized a long-term set (LT-set) of 30 words before the test session; at the start of each test trial, a new short-term set (ST-set) of zero to four words was presented. The subjects gave a positive response to test words that were members of the LT-set or the ST-set presented on that trial, and a negative response to words that were not members of either memory set (distractors). Reaction time (RT) to test words from the ST-set increased with ST-set size; however, RT to LT-set words and distractor words did not depend on the size of the ST-set.

The model proposed by Wescourt and Atkinson (1973; see also Atkinson \& Juola, 1974; Atkinson \& Wescourt, 1975) to account for their results assumes that performance in this task reflects a mixture of two kinds of processing. On some trials, subjects are able to decide whether a test word is a distractor or a member of the positive set simply by evaluating the activity level (called "familiarity" by Atkinson \& Juola, 1974) of the test word's representation in lexical memory. In general, the familiarity of a word is an increasing function of its frequency and recency of presentation; therefore, words with very high and very low familiarity values can be classified as positive items and distractors, respectively. However, when familiarity values fall in an intermediate range, they do not reliably distinguish between distractors and members of the positive set; in such cases a search is conducted to determine whether or not the test word is contained in the representation of the positive set in memory. An assumption of the Wescourt-Atkinson model is that, when a memory search

This research was supported by a grant from the National Institute of Mental Health (MH-21747) to Richard C. Atkinson, who sponsors this paper and takes full editorial responsibility for it. Send requests for reprints to Richard C. Mohs, Department of Psychology, Stanford University, Stanford, California 94305. is executed, searches of STS and LTS are conducted in parallel rather than in sequence. It is further assumed that the time required to determine that an item is in the positive set depends only on the time necessary to search the store where the test item is located; the time to determine that an item is a distractor depends on the slower search (the search of LTS in most cases) since both searches must be completed to determine that the test item is not in the positive set. Subsequent experiments have used both words and digits in the ST-sets (Mohs, Wescourt, \& Atkinson, 1973) and have investigated RT to words that were members of both the ST-set and the LT-set (Mohs \& Atkinson, 1974). The results of both subsequent experiments are consistent with the Wescourt-Atkinson parallel search model; that is, effects of ST-set size were found only for conditions that allowed a response decision immediately following the search of STS.

The present experiment was designed to investigate recognition memory processes involved in processing multiple test probes. The subjects memorized an LT-set of 24 words prior to testing. The ST-sets used did not vary in size from trial to trial as in previous experiments; rather, a new ST-set of four words was presented at the start of each trial. When one test word was presented subjects were to give a positive response if it was a member of the LT-set or the ST-set presented on that trial, and were to give a negative response if the test word was not a member of either memory set. When two test words were presented subjects were to give a positive response only if both words were memory set members; if one or both words were not in the memory sets, subjects were to give a negative response.

Since ST-set size was not a variable, the present experiment does not provide a test of the parallel search model. Rather, the chief purpose in this experiment was to compare the processing of single and double test probes in a task involving both STS and LTS. The paradigm used allows test probes to be drawn from the ST-set or the LT-set; therefore, comparisons can be 
made between processing in STS and LTS as well as comparisons between processing of single and double test probes.

\section{METHOD}

The experiment was run on a programmable display system (Imlac Corporation PDS-1) interacting with a PDP-10 time-sharing computer system. Words in the ST-sets and words used as test stimuli were presented in green capital letters (11 characters/in.) on a dark gray CRT screen and were viewed from a distance of approximately 18 in. Subjects made either a yes or a no response on each trial by striking one of two specified keys on a typewriter-like keyboard located in front of the CRT secreen. Sixteen female students at Stanford University served as paid subjects. Each subject participated in two test sessions.

Words for the LT-set, the ST-sets and words used as distractor test stimuli were taken from the Thorndike-Lorge (1944) word list. All words were four to eight letters in length, had two syllables, and had frequency counts of more than 20 per million. Tested ST-set words and distractor words were each presented only once to each subject. Nontested ST-set words were presented either once or twice to each subject; however, ST-set words that were presented twice appeared only once on each of the 2 test days. A single list of 24 words was used as the LT-set for all subjects. None of the 24 LT-set words were used in the ST-sets. The first two and the last two words of the LT-set were the same for all subjects and were not tested. The middle 20 words of the LT-set were randomized separately for each subject.

Twelve blocks of 20 trials each were constructed. On eight of the trials in each block one test word was presented. These trials were divided among three conditions as follows: (a) on two trials an ST-set word was tested (S condition); (b) on two trials an LT-set word was tested ( $\mathrm{L}$ condition); and (c) on four trials a distractor word was tested. Two test words were presented on each of the remaining trials; two of these trials were from each of the following conditions: (a) trials on which two ST-set words were tested (SS condition); (b) trials on which two LT-set words were tested (LL'condition); (c) trials on which an ST-set word and an LT-set word were tested (SL condition); (d) trials on which an ST-set word and a distractor word were tested (SD condition); (e) trials on which an LT-set word and a distractor word were tested (LD condition); and (f) trials on which two distractor words were tested (DD condition). Across the 12 blocks tested ST-set words were drawn equally often from each serial position in the ST-set for the S, SL, and SD condtions. For the SS condition, the two tested words were taken from each possible combination of ST-set positions equally often across the 12 blocks. On each test day each LT-set word was tested three times. Across the 12 blocks each LT-set word appeared either two or three times in each of the conditions that tested one LT-set word per trial (i.e., the L, SL, and LD conditions), and appeared either four or five times in the LL condition. No LT-set word was tested more than once in a block. Within each block trials were presented in a different random order.

On each trial, test words started one character to the right of and either one line above or one line below a fixation point. For each condition that presented one test word $(S, L$, and $D$ conditions) the two test positions were used equally often in each block. For the SL, SD, and LD conditions the two types of test words appeared equally often in the two positions in each block. For the SS condition, the test word taken from the earlier serial position in the ST-set appeared once as the upper test word and once as the lower test word in each block.

Each subject was contacted by phone at least $18 \mathrm{~h}$ prior to the first test session. The subject was given the LT-set and was told to memorize the list so that it could be recalled in correct serial order at the first test session. Test sessions were scheduled for 2 successive days.

All subjects, satisfied a learning criterion for the LT-set by giving both written and oral recalls of the LT-set in correct serial order at the start of the first test session. After completing both recalls correctly the subject was seated at the CRT screen and given instructions about the task. The subject was told that there would be a series of trials and that the following sequence would be followed on each trial: (a) The word "Ready" would appear on the screen; (b) the subject would then press the spacebar on the keyboard to cause a study symbol (an " $S$ " enclosed in asterisks) to appear at the center of the CRT screen; (c) $750 \mathrm{msec}$ later the four ST-set words would appear, one at a time, slightly to the right of the study symbol; (d) each word would remain on the screen for $1 \mathrm{sec}$, there would be $750 \mathrm{msec}$ between words, and after the last word disappeared there would be a $2.5-\mathrm{sec}$ pause during which the subject was to rehearse the ST-set subvocally; (e) at the end of the pause a fixation point (an asterisk) would appear at the center of the screen for $1 \mathrm{sec}$; (f) then either one or two test words would appear to the right of the fixation point; (g) the test words would remain visible until the subject made a ves or a no response by pressing either the "M" or the "C" key on the keyboard; and (h) a feedback message indicating whether the response was correct or not would appear and remain visible for $2.5 \mathrm{sec}$. The subject was told that, if only one test word was presented, she was to respond yes if the word was a member of the current ST-set or the LT-set: if the word was in neither set she was to respond no. The subject was told that. if two test words appeared. she was to respond yes only if both words were memory set members; if one or both words were not from the ST-set or the LT-set, the subject was to respond no. The experimenter then discussed some sample trials from various conditions and explained the appropriate responses. The instructions emphasized that the subject was to respond as quickly as possible while trying to avoid errors. The keys "M" and " $C$ " were paired with responses so that each subject made positive responses with her preferred hand. The subjects received about 2-3 trials per minute. In addition, there was one $30-\mathrm{sec}$ rest period after the first 60 trials in each session.

When the experimenter was sure that the subject understood the task, 12 practice trials were given. On each practice trial an ST-set of four digits was followed by either one or two test digits. When one test digit was presented, the subject was to respond yes if it was an ST-set member and respond no otherwise: when two test digits were presented, the subject was to respond yes only if both were ST-set members and respond no otherwise. Following the practice trials, the instructions were reviewed and six blocks of test trials were given. At the start of the second day's session, the subject was again required to give both written and oral recalls of the LT-set. The instructions were then reviewed and the subject was given another set of 12 practice trials and the remaining six blocks of test trials.

\section{RESULTS AND DISCUSSION}

Table 1 presents a summary of the results. The letters $S$, L, and D used in the labels at the left of Table 1 represent a probe from the ST-set, the LT-set and a distractor probe, respectively. The label SL, for example, designates the condition that presented an ST-set word and an LT-set word as test stimuli, whereas the D label designates the condition that presented a single distractor word as the test stimulus. For each subject, the mean RT for each condition was calculated. 
Table 1

Mean and Standard Deviation of the Subject Means, Number of Observations, and Percentage Errors for Each Condition

\begin{tabular}{ccccc}
\hline Condition & $\begin{array}{c}\text { RT } \\
\text { (Msec) }\end{array}$ & $\begin{array}{c}\text { SD } \\
\text { (Msec) }\end{array}$ & $\begin{array}{c}\text { Number } \\
\text { of Obser- } \\
\text { vations }\end{array}$ & $\begin{array}{c}\text { Percen- } \\
\text { tage } \\
\text { Errors }\end{array}$ \\
\hline S & 836 & 256 & 384 & 1.6 \\
L & 1037 & 301 & 384 & 5.3 \\
SS & 1101 & 415 & 384 & 1.1 \\
LL & 1234 & 404 & 384 & 4.5 \\
SL & 1359 & 486 & 384 & 5.7 \\
D & 1091 & 568 & 768 & 2.2 \\
DD & 1194 & 512 & 384 & .5 \\
SD & 1419 & 671 & 384 & 5.2 \\
LD & 1580 & 804 & 384 & 6.2 \\
\hline
\end{tabular}

Presented in Table 1 are the mean and standard deviation of the subject means, the total number of observations and the percent errors for each condition. Trials on which an incorrect response was made were not included in the analysis of the RTs.

A two-iactor analysis of variance using subject means as scores was performed on the data from the S, SS, L, and LL conditions; factors included in the analysis were number of probes (one vs. two) and memory set tested (ST-set vs. LT-set). The analysis showed that there were significant effects due to memory set tested, $F(1,15)=$ $40.17, \mathrm{p}<.001$, number of probes, $F(1,15)=32.55$, $p<.001$, and the interaction of these two factors, $F(1,15)=4.70, p<.05$. From Table 1 it is clear that (as in previous experiments using this paradigm, Mohs \& Atkinson, 1974; Mohs et al., 1973; Wescourt \& Atkinson, 1973) probes from the ST-set were recognized more quickly than probes from the LT-set. In addition, for test words from the ST-set and for test words from the LT-set, RT to two probes was greater than RT to a single probe.

One interpretation of these results is simply that two probes are processed sequentially in this task. Adding the assumption that two probes are processed sequentially to the Wescourt-Atkinson Model suggests that, when two probes are presented, subjects: (a) encode one of the probes and evaluate its familiarity; (b) if its familiarity is very low a negative response is executed immediately; (c) if its familiarity is very high the word is judged to be a member of the positive set (since high familiarity indicates a probable member of the positive set): (d) if its familiarity is intermediate representations of the ST-set and the LT-set are searched in parallel for the test word; and (e) if either (c) or (d) indicates that the first word is a member of the positive set, the second word is encoded and processed. A negative response is executed immediately if either the familiarity check or the memory search indicates that one of the probes is a distractor; a positive response is executed only after it is determined that both probes are members of the positive set.
The fact that two positive items were processed more slowly than a single positive item is clearly consistent with the model proposed above. In its simplest form, however, the model does not account for all aspects of the data from positive trials. A first problem is that the difference between the $\mathrm{L}$ and $\mathrm{LL}$ conditions was smaller than the difference between the S and SS conditions; this presents a problem because we have assumed that a search of LTS takes longer than a search of STS. A second (and related) problem is that a paired t test indicated that mean RT for the SL condition was greater than mean RT for the LL condition, $\mathrm{t}(15)=3.49$, $p<.005$. A modification of the model suggested by these results is that finding a test item in LTS facilitates processing of a second item from the LT-set. For example, it could be that, when the first probe is found in the memory structure for the LT-set, a "pointer" is left which temporarily enables very rapid access to the representation of the LT-set in memory. In this regard we should note that a facilitation effect for LT-set words was also found in the Wescourt-Atkinson (1973) study. In that study processing of an LT-set word was faster if an LT-set word was presented on the preceding trial than if either an ST-set word or a distractor word was presented; however, no facilitation across trials was found for ST-set words or for distractors.

A second analysis of variance was performed on the data from the conditions that required a negative response (i.e., the $\mathrm{D}, \mathrm{DD}, \mathrm{SD}$, and $\mathrm{LD}$ conditions). Results of the overall analysis showed that there were significant differences among conditions, $F(3,45)=$ $29.10, p<.001$. A series of orthogonal contrasts suggested by the theoretical analysis given above were then performed.

A contrast of the D and DD conditions with the SD and $L D$ conditions was highly significant, $F(1,45)=$ $76.3, \mathrm{p}<.001$, and accounted for $87.4 \%$ of the total variance for negative trials. A second contrast showed that the difference between the D and DD conditions was only marginally significant, $F(1,45)=3.18$, $.05<\mathrm{p}<.10$. These results are consistent with the view that both items in a two item display are likely to be processed only if a member of the positive set is included in the display. The fact that the difference between the $\mathrm{D}$ and $\mathrm{DD}$ conditions was relatively small supports the assumption that a negative response is executed immediately when either probe in a two-item display is found to be a distractor. We might, however, expect responses in the DD condition to be slightly slower than responses in the $\mathrm{D}$ condition because of the additional time required in the DD condition to decide which of the two test items to process first. A final contrast showed that the difference between the SD and LD conditions was significant, $F(1,45)=7.76, p<.01$. This result was expected since a search of LTS is assumed to require more time than a search of STS. 
Additional support for the hypothesis that two probes are processed sequentially in this task comes from the display order effects found for the SD, LD, and SS conditions. In both the SD and LD conditions mean RT was over $100 \mathrm{msec}$ faster when the distractor word was the bottom word in the test display than when it was the top word in the display. This result suggests that the bottom test word was processed first on most trials; that is, the distractor word was processed first more often when it was at the bottom of the test display than when it was at the top. In the SS condition, mean RT was $1003 \mathrm{msec}$ when the arrangement of words in the display from bottom to top was the same as the order of the words in the ST-set; when top to bottom arrangement was the same as in the ST-set mean RT was $1200 \mathrm{msec}$. Since we have not presented a model for search processes occurring within STS a complete explanation of this result will not be offered. However, this order effect (which is similar to an effect obtained by Baumgarte $\&$ DeRosa, 1973, using double probes in a short-term recognition task) is consistent with the hypothesis that subjects tended to process probes from bottom to top in this task.

\section{REFERENCES}

Atkinson, R. C., \& Juola, J. F. Search and decision processes in recognition memory. In D. H. Krantz, R. C. Atkinson, \& $\mathbf{P}$ Suppes (Eds.), Contemporary developments in mathematical psychology. San Francisco: W. H. Freeman, 1974

Atkinson, R. C., \& Wescourt, K. T. Some remarks on a theory of memory. In P Rabbitt \& S. Dornic (Eds.), Attention and performance. V. London: Academic Press, 1975, in press.

Baumgarte, R., \& DeRosa, D. V. Information processing in a two-item classification task: Relationships among items in a memorized set. Journal of Experimental Psychology, 1973, $100,1-7$.

Mohs, R. C., \& Atkinson, R. C. Recognition time for words in short-term long-term or both memory stores. Journal of Experimental Psy chology, 1974, 102, 830-835.

Mohs, R. C., Wescourt, K. T., \& Atkinson, R. C. Effects of short-term memory contents on short-and long-term memory searches. Memory \& Cognition, 1973, 1, 443-448.

Sternberg, S. Memory scanning: Mental processes revealed by reaction time experiments. American Scientist, 1969, 57, $421-457$.

Thorndike. E. L., \& Lorge, I. The teachers word book of 30,000 words. New York: Columbia University, Teachers College, Bureau of Publications, 1944.

Wescourt, K. T., \& Atkinson, R. C. Scanning for information in short- and long-term memory. Journal of Experimental Psychology, 1973, 98, 95-101.

(Received for publication December 26, 1974.) 